1 Hacettepe Journal of Mathematics and Statistics

$\bigcap$ Volume 47 (5) (2018), 1094-1101

\title{
On almost 2-normed vector lattices
}

\author{
Bahri Turan*† and Fatma Bilici ${ }^{\dagger}$
}

\begin{abstract}
In this paper, we discuss the transformation of a normed vector lattice definition into a 2-normed vector lattice definition and examine the definition of $n$-Banach lattice given by Sağır and Güngör in [A note on n-Banach lattices, J. Appl. Funct. Anal., 2015]. We also give definitions of an almost 2-normed vector lattice and an almost 2-Banach lattice. Then, some theoretical examples and results are given.
\end{abstract}

Keywords: Normed vector lattice, $n$-normed space, almost 2-normed vector lattice, almost 2-Banach lattice.

Mathematics Subject Classification (2010): Primary 46B20, 46B42, 46B99; Secondary $46 \mathrm{~A} 20$

Received: 13.12.2016 Accepted: 15.06.2017 Doi : 10.15672/HJMS.2017.489

\section{Introduction}

All vector spaces are considered over the reals only. Let $(E, \leq)$ be an ordered vector space. A vector $x$ in an ordered vector space $E$ is called positive whenever $0 \leq x$. The set of all positive vectors will be denoted by $E^{+}$. A vector lattice (or a Riesz space) is an ordered vector space $E$ with the additional property that for each pair of element $x, y \in E$ the supremum and infimum of the set $\{x, y\}$ both exist in $E$. For any vector $x$ in a Riesz space $E$, define $x^{+}:=x \vee 0=\sup \{x, 0\}, x^{-}:=-x \vee 0=\sup \{-x, 0\},|x|:=\sup \{x,-x\}$. A Riesz space $E$ is called Archimedean if and only if, given $x, y \in E^{+}$such that $n x \leq y$ holds for all $n=1,2, \ldots$, we have $x=0$. A norm $\|\cdot\|$ on a vector lattice is said to be a lattice norm whenever $|x| \leq|y|$ implies $\|x\| \leq\|y\|$. A vector lattice equipped with a lattice norm is said to be a normed vector lattice. If a normed vector lattice is also norm complete, it is referred as a Banach lattice. Every positive operator from a Banach lattice to a normed vector lattice is continuous [1, Theorem 12.3]. All lattice norms that make

\footnotetext{
*Department of Mathematics, Faculty of Science, Gazi University 06500 Teknikokullar Ankara Turkey, Email: bturan@gazi.edu.tr

$\dagger_{\text {Corresponding Author. }}$

†Department of Mathematics, Faculty of Science, Gazi University 06500 Teknikokullar Ankara Turkey, Email: fatmabilici@gmail.com
} 
a vector lattice a Banach lattice are equivalent [1, Corollary 12.4]. We refer to $[1,6,8,9]$ for definitions and notation not explained here.

The theory of 2-normed spaces was first introduced by Gähler [2] as an interesting linear generalization of the theory of the normed linear spaces which was subsequently studied by many authors. For a fixed number $2 \leq n \in \mathbb{N}$, an $n$-norm on a real vector space $X(\operatorname{dim}(X) \geq n)$ is a mapping $\|\cdot, \ldots, \cdot\|: X^{n} \rightarrow \mathbb{R}$ which satisfies the following four conditions:

1. $\left\|x_{1}, \ldots, x_{n}\right\|=0$ if and only if $x_{1}, \ldots, x_{n}$ are linearly dependent,

2. $\|\cdot, \ldots, \cdot\|$ is invariant under permutation,

3. $\left\|x_{1}, \ldots, x_{n-1}, \alpha x_{n}\right\|=|\alpha|\left\|x_{1}, \ldots, x_{n-1}, x_{n}\right\|$ for any $\alpha \in \mathbb{R}$,

4. $\left\|x_{1}, \ldots, x_{n-1}, y+z\right\| \leq\left\|x_{1}, \ldots, x_{n-1}, y\right\|+\left\|x_{1}, \ldots, x_{n-1}, z\right\|$.

The pair $(X,\|\cdot, \ldots, \cdot\|)$ is called an $n$-normed space. A sequence $\left(x_{k}\right)$ in an $n$-normed space $(X,\|\cdot, \ldots, \cdot\|)$ is said to converge to an $x \in X$ if

$$
\lim _{k \rightarrow \infty}\left\|x_{k}-x, y_{1}, \ldots, y_{n-1}\right\|=0
$$

for all $y_{1}, \ldots, y_{n-1} \in X$.

The following definition of an $n$-lattice norm was introduced by Sağır and Güngör [7] and by the help of this definition they studied spaces of regular operators and order bounded operators between $n$-Banach lattices and Banach lattices. The definition that they gave is as follows.

1.1. Definition. (Definition 1, [7]) Let $E$ be a vector lattice. An $n$-norm $\|\cdot, \ldots, \cdot\|$ on a vector lattice $E$ is said to be an $n$-lattice norm whenever $|x| \leq|y|$ implies

$\left\|x, z_{1}, \ldots, z_{n-1}\right\| \leq\left\|y, z_{1}, \ldots, z_{n-1}\right\|$ for all $z_{1}, \ldots, z_{n-1} \in X$. When $E$ is equipped with an $n$-lattice norm, it is defined as an $n$-normed vector lattice. If the $n$-normed vector lattice $E$ is complete, then it is called as $n$-Banach lattice.

It's known that the space $\ell_{p}$ with $1 \leq p<\infty$ is a vector lattice with respect to the partial ordering introduced by defining that $x \leq y$ iff $x_{k} \leq y_{k}$ holds for all $k$ [1] and also it is an $n$-normed space [3] with the norm

$$
\left\|x_{1}, \ldots, x_{n}\right\|_{p}=\left[\frac{1}{n !} \sum_{j_{1}} \cdots \sum_{j_{n}}\left|\operatorname{det}\left(x_{i j_{k}}\right)\right|^{p}\right]^{\frac{1}{p}}
$$

where $i, k=1,2, \ldots, n, j_{k}=1,2, \ldots$ and

$$
\operatorname{det}\left(x_{i j_{k}}\right)=\operatorname{det}\left(\begin{array}{ccc}
x_{1 j_{1}} & \ldots & x_{1 j_{n}} \\
x_{2 j_{1}} & \ldots & x_{2 j_{n}} \\
\vdots & \ddots & \vdots \\
x_{n j_{1}} & \ldots & x_{n j_{n}}
\end{array}\right) \text {. }
$$

$\left(\ell_{p},\|\cdot, \ldots, \cdot\|_{p}\right)$ was given as an example of $n$-normed vector lattice in [7]. But unfortunately, any vector lattice providing the Definition 1.1 does not exist. Because of this reason, this example is incorrect and this study is meaningless. In this paper, we show the non-existence of such vector lattice providing Definition 1.1 and the incorrectness of this example. Then, we obtain some results by giving a different definition.

\section{2-Norms on vector lattices}

For convenience we will take $n=2$ throughout the study. However, it can be given for an arbitrary $n \in \mathbb{N}, n \geq 2$.

Firstly, we show that the vector lattice $\left(\ell_{p},\|\cdot, \cdot\|_{p}\right)$ is not a 2-normed lattice as given in Definition 1.1. That is why, the example given by Sağır and Güngör is not correct. 
We assume that $\left(\ell_{p},\|\cdot, \cdot\|_{p}\right)$ is 2-normed lattice. If we take $x=(1,1,0,0, \ldots), y=$ $(2,1,0,0, \ldots)$ and $z=y$ then it is easy to see that $x, y, z \in \ell_{p},|x| \leq|y|$ and $1 / 2^{1 / p}=$ $\|x, z\|_{p} \leq\|y, z\|_{p}=0$. This is a contradiction. Even though $z$ and $y$ are chosen as linearly independent, there is still a contradiction. To illustrate, put $x=(2,1,0,0, \ldots)$, $y=(3,4,0,0, \ldots), z=(2,3,0,0, \ldots)$ and note that $x, y, z \in \ell_{p},|x| \leq|y|$ and $4 / 2^{1 / p}=$ $\|x, z\|_{p} \leq\|y, z\|_{p}=1 / 2^{1 / p}$.

Now, we show that not only for this example but also for any vector lattice Definition 1.1 is not provided.

2.1. Lemma. If $(E,\|\cdot, \cdot\|)$ is a 2-normed vector lattice, there exists an element $x$ in $E$ such that $x^{+} \neq 0$ and $x^{-} \neq 0$.

Proof. Every 2-normed vector lattice is Archimedean. Let $x, y \in E^{+}$be such that $n x \leq y$ holds for $n \in \mathbb{N}$. Then $\|n x, z\| \leq\|y, z\|$ for all $z \in E, n \in \mathbb{N}$. By the definition of 2-lattice norm, $\|x, z\| \leq(1 / n)\|y, z\|$ for all $z \in E, n \in \mathbb{N}$. Since $\mathbb{R}$ is Archimedean, $\|x, z\|=0$ for all $z \in E$. This means $x=0$. Assume by the way of contradiction that $(x-y)^{+}=0$ or $(x-y)^{-}=0$ for all $x, y \in E$. Then $x \leq y$ or $y \leq x$ and so $E$ is totally ordered. From these, $E$ is totally ordered and Archimedean. By the Proposition 3.4 in [8, Chapter II] $E$ is isomorphic to $\mathbb{R}$, which is impossible. Hence the statement is proven.

2.2. Theorem. Let $E$ be a vector lattice, then there is not any 2-norm on $E$ such that $E$ is 2-normed vector lattice.

Proof. Suppose there is a 2-norm $\|\cdot, \cdot\|$ on $E$ such that whenever $|x| \leq|y|$ implies $\|x, z\| \leq\|y, z\|$ for all $z \in E$. By Lemma 2.1 there exists an element $x$ in E, different from zero, such that $x^{+} \neq 0$ and $x^{-} \neq 0$. As $x^{+} \wedge x^{-}=0, x^{+}$and $x^{-}$are linearly independent [1, Chapter 1.1, Exercises 2]. Since $\left|x^{+}\right|=x^{+} \leq x^{+}+x^{-}=\left|x^{+}+x^{-}\right|$and $E$ is a 2-normed vector lattice, then $\left\|x^{+}, x^{+}+x^{-}\right\| \leq\left\|x^{+}+x^{-}, x^{+}+x^{-}\right\|=0$. This contradicts that $x^{+}$and $x^{+}+x^{-}$are linearly independent.

As seen from the above theorem there is not any vector lattice that provide the definition given by Sağır and Güngör. So, we give the following definition.

2.3. Definition. A norm $\|\cdot\|$ on a vector lattice $E$ is said to be an almost lattice norm if there is a constant $K>0$ such that whenever for all $x, y \in E,|x| \leq|y|$ implies $\|x\| \leq K\|y\|$. A vector lattice equipped with an almost lattice norm is said to be an almost normed vector lattice. If an almost normed vector lattice is also norm complete, it is referred as an almost Banach lattice.

2.4. Theorem. Every positive operator from an almost Banach lattice to an almost normed vector lattice is continuous.

Proof. The proof is the same as the proof of Theorem 12.3 in [1] with a slight difference.

2.5. Corollary. All almost lattice norms that make a vector lattice an almost Banach lattice are equivalent.

Proof. It is the same as the proof of Corollary 12.4 in [1].

Let $(E,\|\cdot, \cdot\|)$ be a 2 -normed space and $\{a, b\}$ be a linearly independent set on $E$. The following functions

$$
\begin{aligned}
\|x\|^{*} & =\|x, a\|+\|x, b\| \quad(\text { see }[2]) \\
\|x\|_{*} & =\max \{\|x, a\|,\|x, b\|\} \quad(\operatorname{see}[4])
\end{aligned}
$$


define a norm which is called as derived norm on E. Hence, all 2-normed spaces are normed spaces. It is easily seen that the norms $\|\cdot\|^{*}$ and $\|\cdot\|_{*}$ are equivalent. Therefore, it is not important which one is taken in the following definition.

2.6. Definition. Let $E$ be a vector lattice, $\|\cdot, \cdot\|$ be a 2 -norm on $E$ and $\|\cdot\|^{*}$ be derived norm defined with respect to the linearly independent set $\{a, b\}$. The 2 -norm $\|\cdot, \cdot\|$ is said to be an almost 2-lattice norm with respect to $\{a, b\}$ if the norm $\|\cdot\|^{*}$ is almost lattice norm. The 2-norm $\|\cdot, \cdot\|$ is said to be an almost 2-lattice norm whenever the norm $\|\cdot\|^{*}$ is an almost lattice norm with respect to all linearly independent sets $\{a, b\}$ in $E$. When $E$ is equipped with an almost 2-lattice norm, it is defined as an almost 2-normed vector lattice. If an almost 2-normed vector lattice $E$ is complete with the $\|\cdot\|^{*}$ norm with respect to all linearly independent sets $\{a, b\}$ in $E$, then it's called as an almost 2-Banach lattice.

By Corollary 2.5, if $E$ is an almost 2-Banach lattice then the derived norms $\|\cdot\|^{*}$ are equivalent for all linearly independent sets $\{a, b\}$ in $E$.

2.7. Example. Let $E=\mathbb{R}^{2}$ equipped with Euclidean 2-norm $\|\cdot, \cdot\|$ defined by

$$
\|x, y\|=\left|\operatorname{det}\left(\begin{array}{ll}
x_{1} & y_{1} \\
x_{2} & y_{2}
\end{array}\right)\right|=\left|x_{1} y_{2}-x_{2} y_{1}\right|
$$

where $x=\left(x_{1}, x_{2}\right), y=\left(y_{1}, y_{2}\right) \in \mathbb{R}^{2}$. This is the area of the parallelogram determined by the vectors $x$ and $y$. Given the standard basis $\left\{e_{1}, e_{2}\right\}$, define a norm by

$$
\|x\|^{*}=\left\|x, e_{1}\right\|+\left\|x, e_{2}\right\|
$$

where $x \in \mathbb{R}^{2}$. Choose $x=\left(x_{1}, x_{2}\right), y=\left(y_{1}, y_{2}\right) \in \mathbb{R}^{2}$ with $|x| \leq|y|$. We have

$$
\|x\|^{*}=\left\|x, e_{1}\right\|+\left\|x, e_{2}\right\|=\left|x_{1}\right|+\left|x_{2}\right| \leq\left|y_{1}\right|+\left|y_{2}\right|=\left\|y, e_{1}\right\|+\left\|y, e_{2}\right\|=\|y\|^{*} .
$$

If we choose linearly independent set $\{a=(0,1), b=(1,2)\}, x=(1,1), y=(1,2)$ then $|x| \leq|y|$ but not $\|x\|^{*} \leq\|y\|^{*}$. Hence, the norm $\|\cdot\|^{*}$ defined with respect to linearly independent set $\{a=(0,1), b=(1,2)\}$ is not lattice norm. But $\left(\mathbb{R}^{2},\|\cdot, \cdot\|\right)$ is almost 2Banach lattice.

2.8. Theorem. $\left(\mathbb{R}^{2},\|\cdot, \cdot\|\right)$ is almost 2 -Banach lattice.

Proof. Choose arbitrary linearly independent set $\left\{a=\left(a_{1}, a_{2}\right), b=\left(b_{1}, b_{2}\right)\right\}$ and $x=$ $\left(x_{1}, x_{2}\right), y=\left(y_{1}, y_{2}\right) \in \mathbb{R}^{2}$ such that $|x| \leq|y|$. We get

$$
\begin{aligned}
\|x\|^{*} & =\|x, a\|+\|x, b\| \\
& =\left|x_{1} a_{2}-x_{2} a_{1}\right|+\left|x_{1} b_{2}-x_{2} b_{1}\right| \\
& \leq\left|x_{1}\right|\left|a_{2}\right|+\left|x_{2}\right|\left|a_{1}\right|+\left|x_{1}\right|\left|b_{2}\right|+\left|x_{2}\right|\left|b_{1}\right| \\
& =\left|x_{1}\right|\left(\left|a_{2}\right|+\left|b_{2}\right|\right)+\left|x_{2}\right|\left(\left|a_{1}\right|+\left|b_{1}\right|\right) \\
& \leq\left(\left|x_{1}\right|+\left|x_{2}\right|\right)\left(\left|a_{1}\right|+\left|a_{2}\right|+\left|b_{1}\right|+\left|b_{2}\right|\right) \\
& \leq\left(\left|y_{1}\right|+\left|y_{2}\right|\right)\left(\left|a_{1}\right|+\left|a_{2}\right|+\left|b_{1}\right|+\left|b_{2}\right|\right)
\end{aligned}
$$


and

$$
\begin{aligned}
\left(\left|y_{1}\right|+\left|y_{2}\right|\right)\|a, b\|= & \left(\left|y_{1}\right|+\left|y_{2}\right|\right)\left(\left|a_{1} b_{2}-a_{2} b_{1}\right|\right) \\
= & \left|y_{1} a_{1} b_{2}-y_{1} a_{2} b_{1}\right|+\left|y_{2} a_{1} b_{2}-y_{2} a_{2} b_{1}\right| \\
= & \left|y_{1} a_{1} b_{2}-y_{2} b_{1} a_{1}+y_{2} b_{1} a_{1}-y_{1} a_{2} b_{1}\right| \\
& +\left|y_{2} a_{1} b_{2}-y_{1} a_{2} b_{2}+y_{1} a_{2} b_{2}-y_{2} a_{2} b_{1}\right| \\
\leq & \left|a_{1}\right|\left(\left|y_{1} b_{2}-y_{2} b_{1}\right|\right)+\left|b_{1}\right|\left(\left|y_{2} a_{1}-y_{1} a_{2}\right|\right) \\
& +\left|b_{2}\right|\left(\left|y_{2} a_{1}-y_{1} a_{2}\right|\right)+\left|a_{2}\right|\left(\left|y_{1} b_{2}-y_{2} b_{1}\right|\right) \\
= & \left(\left|a_{1}\right|+\left|a_{2}\right|\right)\|y, b\|+\left(\left|b_{1}\right|+\left|b_{2}\right|\right)\|y, a\| \\
\leq & \left(\left|a_{1}\right|+\left|a_{2}\right|+\left|b_{1}\right|+\left|b_{2}\right|\right)(\|y, b\|+\|y, a\|) \\
= & \left(\left|a_{1}\right|+\left|a_{2}\right|+\left|b_{1}\right|+\left|b_{2}\right|\right)\|y\|^{*} .
\end{aligned}
$$

These yield that

$$
\begin{aligned}
\|x\|^{*} & \leq\left(\left|y_{1}\right|+\left|y_{2}\right|\right)\left(\left|a_{1}\right|+\left|a_{2}\right|+\left|b_{1}\right|+\left|b_{2}\right|\right) \\
& \leq \frac{\left(\left|a_{1}\right|+\left|a_{2}\right|+\left|b_{1}\right|+\left|b_{2}\right|\right)^{2}}{\|a, b\|}\|y\|^{*} .
\end{aligned}
$$

Hence $\left(\mathbb{R}^{2},\|\cdot, \cdot\|\right)$ is an almost 2-normed vector lattice. Now, we show that $\left(\mathbb{R}^{2},\|\cdot\|^{*}\right)$ is complete. Let $\left(z_{k}\right)=\left(x_{k}, y_{k}\right) \subseteq \mathbb{R}^{2}$ be a $\|\cdot\|^{*}$-Cauchy sequence. By the above inequalities we drive

$$
\left\|z_{k}-z_{t}\right\|_{1}=\left|x_{k}-x_{t}\right|+\left|y_{k}-y_{t}\right| \leq \frac{\left(\left|a_{1}\right|+\left|a_{2}\right|+\left|b_{1}\right|+\left|b_{2}\right|\right)^{2}}{\|a, b\|}\left\|z_{k}-z_{t}\right\|^{*}
$$

and

$$
\left\|z_{k}-z_{t}\right\|^{*} \leq\left(\left|a_{1}\right|+\left|a_{2}\right|+\left|b_{1}\right|+\left|b_{2}\right|\right)\left\|z_{k}-z_{t}\right\|_{1} .
$$

A simple argument shows that $\left(\mathbb{R}^{2},\|\cdot\|^{*}\right)$ is complete because $\left(\mathbb{R}^{2},\|\cdot\|_{1}\right)$ is complete where $\|z\|_{1}=|x|+|y|, z=(x, y)$.

The space of all bounded functions on a non-empty set $X$ is denoted by $B(X)$ and $B(X)$ is a Banach lattice with its usual norm defined by

$$
\|f\|_{\infty}=\sup _{x \in X}|f(x)| \quad(f \in B(X)),
$$

and the pointwise partial order defined by $f \leq g$ when $f(x) \leq g(x)$ for all $x$ in $X$. The following formula define 2-norm on $B(X)$

$$
\|f, g\|_{\infty}:=\sup _{x, y \in X}\left|\operatorname{det}\left(\begin{array}{cc}
f(x) & g(x) \\
f(y) & g(y)
\end{array}\right)\right|=\sup _{x, y \in X}|f(x) g(y)-f(y) g(x)|
$$

2.9. Theorem. $\left(B(X),\|\cdot, \cdot\|_{\infty}\right)$ is an almost 2-Banach lattice.

Proof. Let $f, g \in B(X),|f| \leq|h|$ and $\|\cdot\|^{*}$ be derived norm, defined with respect to arbitrary linearly independent set $\left\{g_{1}, g_{2}\right\}$. We get

$$
\begin{aligned}
\|f\|^{*}= & \left\|f, g_{1}\right\|_{\infty}+\left\|f, g_{2}\right\|_{\infty} \\
= & \sup _{x, y \in X}\left|f(x) g_{1}(y)-f(y) g_{1}(x)\right|+\sup _{x, y \in X}\left|f(x) g_{2}(y)-f(y) g_{2}(x)\right| \\
\leq & \sup _{x, y \in X}|f(x)|\left|g_{1}(y)\right|+\sup _{x, y \in X}|f(y)|\left|g_{1}(x)\right| \\
& +\sup _{x, y \in X}|f(x)|\left|g_{2}(y)\right|+\sup _{x, y \in X}|f(y)|\left|g_{2}(x)\right| \\
\leq & 2\|f\|_{\infty}\left(\left\|g_{1}\right\|_{\infty}+\left\|g_{2}\right\|_{\infty}\right)
\end{aligned}
$$


Since $\|f\|_{\infty} \leq\|h\|_{\infty}$,

$$
\|f\|^{*} \leq 2\|h\|_{\infty}\left(\left\|g_{1}\right\|_{\infty}+\left\|g_{2}\right\|_{\infty}\right)
$$

holds. On the other hand, we have

$$
\begin{aligned}
& \|h\|_{\infty}\left\|g_{1}, g_{2}\right\|_{\infty}=\sup _{z \in X}|h(z)| \sup _{x, y \in X}\left|g_{1}(x) g_{2}(y)-g_{1}(y) g_{2}(x)\right| \\
& =\sup _{z, x, y \in X}\left|h(z) g_{1}(x) g_{2}(y)-h(z) g_{1}(y) g_{2}(x)\right| \\
& =\sup _{z, x, y \in X}\left|\begin{array}{c}
h(z) g_{1}(x) g_{2}(y)-h(y) g_{1}(x) g_{2}(z)+h(y) g_{1}(x) g_{2}(z) \\
-h(x) g_{1}(y) g_{2}(z)+h(x) g_{1}(y) g_{2}(z)-h(z) g_{1}(y) g_{2}(x)
\end{array}\right| \\
& \leq \sup _{z, x, y \in X}\left\{\begin{array}{c}
\left|g_{1}(x)\right|\left|h(z) g_{2}(y)-h(y) g_{2}(z)\right| \\
+\left|g_{2}(z)\right|\left|h(y) g_{1}(x)-h(x) g_{1}(y)\right| \\
+\left|g_{1}(y)\right|\left|h(x) g_{2}(z)-h(z) g_{2}(x)\right|
\end{array}\right\} \\
& \leq\left\|g_{1}\right\|_{\infty}\left\|h, g_{2}\right\|_{\infty}+\left\|g_{2}\right\|_{\infty}\left\|h, g_{1}\right\|_{\infty}+\left\|g_{1}\right\|_{\infty}\left\|h, g_{2}\right\|_{\infty} \\
& =2\left\|g_{1}\right\|_{\infty}\left\|h, g_{2}\right\|_{\infty}+\left\|g_{2}\right\|_{\infty}\left\|h, g_{1}\right\|_{\infty} \\
& \leq 2\left(\left\|g_{1}\right\|_{\infty}+\left\|g_{2}\right\|_{\infty}\right)\left(\left\|h, g_{2}\right\|_{\infty}+\left\|h, g_{1}\right\|_{\infty}\right) \\
& =2\left(\left\|g_{1}\right\|_{\infty}+\left\|g_{2}\right\|_{\infty}\right)\|h\|^{*} \text {. }
\end{aligned}
$$

Accordingly,

$$
\|f\|^{*} \leq 4 \frac{\left(\left\|g_{1}\right\|_{\infty}+\left\|g_{2}\right\|_{\infty}\right)^{2}}{\left\|g_{1}, g_{2}\right\|_{\infty}}\|h\|^{*} .
$$

Hence $B(X)$ is an almost 2-normed vector lattice. Let $\left(f_{k}\right) \subseteq B(X)$ be a $\|\cdot\|^{*}$-Cauchy sequence. By the above inequalities we drive

$$
\left\|f_{k}-f_{t}\right\|_{\infty} \leq \frac{2\left(\left\|g_{1}\right\|_{\infty}+\left\|g_{2}\right\|_{\infty}\right)}{\left\|g_{1}, g_{2}\right\|_{\infty}}\left\|f_{k}-f_{t}\right\|^{*}
$$

and

$$
\left\|f_{k}-f_{t}\right\|^{*} \leq 2\left\|f_{k}-f_{t}\right\|_{\infty}\left(\left\|g_{1}\right\|_{\infty}+\left\|g_{2}\right\|_{\infty}\right) .
$$

A simple argument shows that $\left(B(X),\|\cdot\|^{*}\right)$ is complete because $\left(B(X),\|\cdot\|_{\infty}\right)$ is complete.

As for every subvector lattice of almost 2-normed vector lattice is an almost 2-normed vector lattice and $\left(C(X),\|\cdot\|_{\infty}\right)$ is complete, we can give the following corollary.

2.10. Corollary. If $X$ is a non-empty compact topological space and $C(X)$ is a real linear space of all real continuous functions on $X$, then $C(X)$ is an almost 2-Banach lattice with the same partial ordering and 2-norm given in the Theorem 2.9.

For the special case $X=\mathbb{N}$ we have $B(X)=\ell_{\infty}$. Therefore, we get the following corollary because $\left(\ell_{\infty},\|\cdot\|_{\infty}\right)$ is complete.

2.11. Corollary. $\ell_{\infty}$ is an almost 2-Banach lattice with the same partial ordering and 2-norm given in the Theorem 2.9.

As mentioned in the introduction, the space $\ell_{p}(1 \leq p<\infty)$ is a vector lattice and 2-normed space with the norm

$$
\|x, y\|_{p}=\left(\frac{1}{2} \sum_{j} \sum_{k}\left|\operatorname{det}\left(\begin{array}{ll}
x_{j} & x_{k} \\
y_{j} & y_{k}
\end{array}\right)\right|^{p}\right)^{\frac{1}{p}}
$$

2.12. Theorem. $\left(\ell_{p},\|\cdot, \cdot\|_{p}\right)$ is an almost 2 -Banach lattice. 
Proof. Let $|x| \leq|y|$ and $\|\cdot\|^{*}$ be derived norm defined with respect to arbitrary linearly independent set $\{a, b\}$ in $\ell_{p}$. Because $\|x\|_{p} \leq\|y\|_{p}$ and from Fact 2.1 in [3] it follows that

$$
\begin{aligned}
\|x\|^{*} & =\|x, a\|_{p}+\|x, b\|_{p} \\
& \leq 2^{1-\frac{1}{p}}\|x\|_{p}\|a\|_{p}+2^{1-\frac{1}{p}}\|x\|_{p}\|b\|_{p} \\
& =2^{1-\frac{1}{p}}\left(\|a\|_{p}+\|b\|_{p}\right)\|x\|_{p} \\
& \leq 2^{1-\frac{1}{p}}\left(\|a\|_{p}+\|b\|_{p}\right)\|y\|_{p} .
\end{aligned}
$$

From Lemma 2.2 in [5] we have

$$
\|y\|_{p}\|a, b\|_{p} \leq 2\|a\|_{p}\|y, b\|_{p}+\|b\|_{p}\|y, a\|_{p} \leq 2\left(\|a\|_{p}+\|b\|_{p}\right)\|y\|^{*} .
$$

Then,

$$
\|x\|^{*} \leq \frac{2^{2-\frac{1}{p}}}{\|a, b\|_{p}}\left(\|a\|_{p}+\|b\|_{p}\right)^{2}\|y\|^{*} .
$$

On the other hand, from the above inequalities, we obtain

$$
\|x\|^{*} \leq 2^{1-\frac{1}{p}}\left(\|a\|_{p}+\|b\|_{p}\right)\|x\|_{p} \text { and }\|x\|_{p} \leq \frac{2\left(\|a\|_{p}+\|b\|_{p}\right)}{\|a, b\|_{p}}\|x\|^{*}
$$

for all $x \in \ell_{p}$. Since $\left(\ell_{p},\|\cdot\|_{p}\right)$ is complete, we may conclude $\left(\ell_{p},\|\cdot, \cdot\|_{p}\right)$ is an almost Banach lattice.

As mentioned above, each 2-normed space is a normed space with the norm $\|\cdot\|^{*}$. It is easily see that a convergent sequence in the 2-norm is convergent sequence in the $\|\cdot\|^{*}$ norm. The converse have not yet been concluded exactly. Gunawan and Mashadi show that a convergent sequence in the $\|\cdot\|^{*}$ norm is a convergent sequence in the 2-norm in finite dimensional 2-normed space and $\ell_{p}$ space [4]. We obtain that it is hold in an almost 2-Banach lattice.

2.13. Theorem. Let $(E,\|\cdot, \cdot\|)$ be an almost 2 -Banach lattice and $\|\cdot\|^{*}$ be a norm defined with respect to arbitrary linearly independent set $\{a, b\}$. A sequence in $E$ is convergent in the $\|\cdot\|^{*}$ norm if and only if it is convergent in the $\|\cdot, \cdot\| 2$-norm.

Proof. If a sequence in $\mathrm{X}$ is convergent in the 2-norm, then it will certainly be convergent in the $\|\cdot\|^{*}$ norm. Conversely, suppose that $\left(x_{k}\right)$ converges to an $x$ in the $\|\cdot\|^{*}$ norm and let $y$ be an arbitrary element of $E$. Since dimension of $E$ is greater than 1 , there exists an element $z \in E$ such that $y, z$ are linearly independent. Let $\|\cdot\|^{\boldsymbol{\Delta}}$ be the derived norm, defined with respect to linearly independent set $\{y, z\}$. If $E$ is an almost 2-Banach lattice, then $\|\cdot\|^{*}$ and $\|\cdot\|^{\wedge}$ are equivalent by Corollary 2.5 . Then, we have

$$
0 \leq\left\|x_{k}-x, y\right\| \leq\left\|x_{k}-x, y\right\|+\left\|x_{k}-x, z\right\|=\left\|x_{k}-x\right\|^{\mathbf{\Delta}} \leq M\left\|x_{k}-x\right\|^{*}
$$

for some $M>0$. This implies that $\left(x_{k}\right)$ converges to $x$ in the $\|\cdot, \cdot\|$ norm.

We can give the following corollary from the above theorem.

2.14. Corollary. A sequence $\left(f_{k}\right)$ in $B(X)$ is convergent in the $\|\cdot\|^{*}$ norm if and only if it is convergent in the $\|\cdot, \cdot\|_{\infty} 2$-norm.

2.15. Theorem. Let $\left(E,\|\cdot, \cdot\|_{1}\right)$ and $\left(E,\|\cdot, \cdot\|_{2}\right)$ be almost 2-Banach lattices. A sequence $\left(x_{k}\right)$ in $E$ is convergent in the $\|\cdot, \cdot\|_{1} 2$-norm if and only if it is convergent in the $\|\cdot, \cdot\|_{2}$ 2-norm. 
Proof. Let $\|\cdot\|_{1}^{*}$ and $\|\cdot\|_{2}^{*}$ be derived norms, defined with respect to any linearly independent set $\{a, b\}$. Choose $\left(x_{k}\right) \subseteq E, x \in E$ and suppose that $\left(x_{k}\right)$ converges to $x$ in the $\|\cdot, \cdot\|_{1} 2$-norm, that is $\lim _{k \rightarrow \infty}\left\|x_{k}-x, y\right\|_{1}=0$ for every $y \in E$. Hence $\lim _{k \rightarrow \infty}\left\|x_{k}-x\right\|_{1}^{*}=0$, by the Theorem 2.13. Since $\left(E,\|\cdot, \cdot\|_{1}\right)$ and $\left(E,\|\cdot, \cdot\|_{2}\right)$ are almost 2-Banach lattice, $\|\cdot\|_{1}^{*}$ and $\|\cdot\|_{2}^{*}$ are equivalent. Therefore, $\lim _{k \rightarrow \infty}\left\|x_{k}-x\right\|_{2}^{*}=0$, so by the Theorem 2.13 $\lim _{k \rightarrow \infty}\left\|x_{k}-x, y\right\|_{2}=0$ for every $y \in E$. The proof of the converse direction is similar.

\section{References}

[1] Aliprantis, C.D. and Burkinshaw, O. Positive operators, Academic Press, Orlando, 1985.

[2] Gähler, S. Lineare 2-normierte räume, Math. Nachr. 28, 1-43, 1964.

[3] Gunawan, H. The space of p-summable sequences and its natural n-norms, Bull. Austral. Math. Soc. 64, 137-147, 2001.

[4] Gunawan, H. and Mashadi, M. On n-normed spaces, Int. J. Math. Math. Sci. 27, 631-639, 2001.

[5] Idris, M., Ekariani, S. and Gunawan, H. On the space of p-summable sequences, Mat. Vesnik. 65, 58-63, 2013.

[6] Luxemburg, W. A. J. and Zaanen, A. C. Riesz spaces I, North-Holland, Amsterdam, 1971.

[7] Sağır, B. and Güngör, N. A note on n-Banach lattices, J. Appl. Funct. Anal. 10, 70-77, 2015.

[8] Schaefer, H.H. Banach lattices and positive operators, Springer, Grundlehren 215, Berlin, 1974.

[9] Zaanen, A. C. Riesz spaces II, North-Holland, Amsterdam, 1983. 